\title{
Four-loop quark mass and field renormalization in the on-shell scheme
}

\section{Peter Marquard*广}

Deutsches Elektronen-Synchrotron, DESY 15738 Zeuthen, Germany

E-mail: peter.marquard@desy.de

\author{
Alexander V. Smirnov \\ Research Computing Center, Moscow State University \\ 119991, Moscow, Russia

\section{Vladimir A. Smirnov} \\ Skobeltsyn Institute of Nuclear Physics of Moscow State University \\ 119991, Moscow, Russia

\section{Matthias Steinhauser} \\ Institut für Theoretische Teilchenphysik, Karlsruhe Institute of Technology (KIT) \\ 76128 Karlsruhe, Germany
}

We review recent results for the on-shell renormalization constants of QCD and the anomalous magnetic moment of the muon. These two quantities are related since they rely on the same class of Feynman integrals, so-called on-shell integrals.

13th International Symposium on Radiative Corrections (Applications of Quantum Field Theory to Phenomenology)

25-29 September, 2017

St. Gilgen, Austria

* Speaker.

${ }^{\dagger}$ DESY 18-035 


\section{Introduction}

On-shell Feynman integrals play an important role in many physical applications. In the following we present an overview of recently obtained results involving this class of integrals. In particular, we present results for the $\overline{\mathrm{MS}}$-on-shell relation, the heavy-quark wave function renormalization constant in the on-shell scheme and the anomalous magnetic moment of the muon. In the following we briefly review the calculational aspects and present our results.

\section{Computational aspects}

In both cases, the calculation of the on-shell renormalization constants in QCD and the anomalous magnetic moment of the muon, we follow the same approach. The diagrams are generated using qgraf [1] and are then processed by q2e/exp [2,3] and FORM [4, 5]. To avoid tensor integrals in both cases, suitable projectors are applied. The appearing scalar integrals are then reduced to a small set of master integrals using FIRE [6, 7, 8] and Crusher [9]. To obtain a minimal set of master integrals we employ the help of tsort which is part of recent FIRE releases. The main obstacle is the calculation of these master integrals. To obtain them we make use of a variety of techniques. The most simple ones can be calculated in terms of Gamma functions. For the more complicated ones we can derive Mellin-Barnes representations which can be evaluated with high accuracy. For the most complicated ones we employ the method of sector decomposition implemented in FIESTA $[10,11,12]$. Combining these techniques we have been able to obtain results which are suitable for phenomenological applications. We refrain from presenting any more details here but refer the reader to the extended discussion in Ref. [13].

\section{On-shell renormalization constants}

The renormalized quark propagator is given by

$$
\begin{gathered}
S_{F}(q)=\frac{-i Z_{2}^{\mathrm{OS}}}{q-m^{0}+\Sigma(q, M)} \\
\stackrel{q^{2} \rightarrow M^{2}}{\longrightarrow} \frac{-i}{q-M},
\end{gathered}
$$

where the renormalization constants are defined as

$$
\begin{aligned}
& m^{0}=Z_{m}^{\mathrm{OS}} M, \\
& \psi_{0}=\sqrt{Z_{2}^{\mathrm{OS}}} \psi .
\end{aligned}
$$

Here, $\psi$ is the quark field with mass $m, M$ is the on-shell mass and bare quantities are denoted by a superscript 0 .

\section{1 $\overline{\mathrm{MS}}-$ on-shell relation}

Instead of the renormalization constant $Z_{2}^{\mathrm{OS}}$, we will present results for the finite quantity

$$
z_{m}(\mu)=\frac{m(\mu)}{M}=\frac{Z_{m}^{\mathrm{OS}}}{Z_{m}^{\overline{\mathrm{MS}}}}
$$


which relates the different renormalization schemes. Note that $z_{m}(\mu)$ depends on $\alpha_{s}(\mu)$ and $\log (\mu / M)$ and has the following perturbative expansion

$$
z_{m}(\mu)=\sum_{n \geq 0}\left(\frac{\alpha_{s}(\mu)}{\pi}\right)^{n} z_{m}^{(n)}(\mu)
$$

For QCD, we obtain for the four-loop coefficient [14]

$$
z_{m}^{(4)}=-3654.15 \pm 1.64+(756.942 \pm 0.040) n_{l}-43.4824 n_{l}^{2}+0.678141 n_{l}^{3} .
$$

where $n_{l}$ denotes the number of light quarks. For the inverse relation

$$
c_{m}(\mu)=\frac{M}{m(\mu)}=\frac{Z_{m}^{\overline{\mathrm{MS}}}}{Z_{m}^{\mathrm{OS}}}
$$

we find

$$
c_{m}^{(4)}=3567.60 \pm 1.64-(745.721 \pm 0.040) n_{l}+43.3963 n_{l}^{2}-0.678141 n_{l}^{3}
$$

To illustrate the numerical impact of the four-loop contribution we present explicit results for top and bottom quarks. For the case of the top quark $\left(n_{l}=5\right)$ they read

$$
\begin{aligned}
m_{t}\left(m_{t}\right) & =M_{t}\left(1-0.4244 \alpha_{s}-0.9246 \alpha_{s}^{2}-2.593 \alpha_{s}^{3}-(8.949 \pm 0.018) \alpha_{s}^{4}\right) \\
& =173.34-7.924-1.859-0.562-(0.209 \pm 0.0004) \mathrm{GeV},
\end{aligned}
$$

while for the bottom quark $\left(n_{l}=4\right)$ we get

$$
\begin{aligned}
M_{b} & =m_{b}\left(m_{b}\right)\left(1+0.4244 \alpha_{s}+0.9401 \alpha_{s}^{2}+3.045 \alpha_{s}^{3}+(12.685 \pm 0.025) \alpha_{s}^{4}\right) \\
& =4.163+0.398+0.199+0.145+(0.136 \pm 0.0003) \mathrm{GeV}
\end{aligned}
$$

In both cases the second line shows the contributions of the different loop orders after multiplying with the corresponding power of $\alpha_{s}$. Since the pole mass suffers from infrared problems the convergence is not very good for the top quark and rather bad for the bottom quark. In the case of the top quark the asymptotics of the series have been discussed in detail in $[15,16]$.

To show an example of a scheme conversion between infrared safe schemes we present our results for the conversion between the potential subtracted (PS) mass [17] and the $\overline{\mathrm{MS}}$ mass

$$
\begin{aligned}
m_{t}^{\mathrm{PS}}\left(\mu_{f}=80 \mathrm{GeV}\right)= & 163.508+(7.531-3.685)+(1.607-0.989) \\
& +(0.495-0.403)+(0.195-0.211 \pm 0.0004) \mathrm{GeV} \\
= & 163.508+3.847+0.618+0.092-(0.016 \pm 0.0004) \mathrm{GeV}
\end{aligned}
$$

Here, infrared unsafe contributions in both the relations between on-shell and PS mass on one side and $\overline{\mathrm{MS}}$ to on-shell mass on the other side cancel. To demonstrate this cancellation we show in the second line both contributions separately. In the sum we obtain a very well convergent series. 


\subsection{Wave-function renormalization constant}

The wave function renormalization constant is phenomenologically of less importance. It will make its first appearance in a physical application in the calculation of on-shell form factors at four loops. It can expanded in a perturbative series

$$
Z_{2}^{\mathrm{OS}}=1+\sum_{j \geq 1}\left(\frac{\alpha_{s}^{0}(\mu)}{\pi}\right)^{j}\left(\frac{e^{\gamma_{E}}}{4 \pi}\right)^{-j \varepsilon}\left(\frac{\mu^{2}}{M^{2}}\right)^{j \varepsilon} \delta Z_{2}^{(j)}
$$

where the bare strong coupling constant $\alpha_{s}^{0}$ has been used for the parametrization. Note that $\delta Z_{2}^{(i)}$ for $i \geq 3$ depend on the QCD gauge parameter $\xi$. Similar to $\alpha_{s}$ we keep in $\delta Z_{2}^{(4)}$ also $\xi$ in its bare form. With these choices we can define the coefficients $\delta Z_{2}^{(i)}$ such that that they do not contain $\log \left(\mu^{2} / M^{2}\right)$ terms. In fact, they can be combined to the factors $\left(\mu^{2} / M^{2}\right)^{i \varepsilon}$ where $i$ is the loop order (cf. Eq. (3.11)). The renormalization of $\alpha_{s}$ and $\xi-1$ is multiplicative and hence $\alpha_{s}^{0}$ and $\xi^{0}$ can be replaced in a straightforward way by their renormalized counterparts using the relations

$$
\begin{aligned}
\alpha_{s}^{0} & =\left(\mu^{2}\right)^{2 \varepsilon} Z_{\alpha_{s}} \alpha_{s}, \\
\xi^{0}-1 & =Z_{3}(\xi-1),
\end{aligned}
$$

where

$$
\begin{aligned}
Z_{\alpha_{s}} & =1+\frac{1}{\varepsilon}\left(\frac{n_{f}}{6}-\frac{11}{12} C_{A}\right) \frac{\alpha_{s}}{\pi}+\ldots \\
Z_{3} & =1+\frac{1}{\varepsilon}\left[-\frac{n_{f}}{6}+\left(\frac{5}{12}+\frac{1}{8} \xi\right) C_{A}\right] \frac{\alpha_{s}}{\pi}+\ldots
\end{aligned}
$$

Since we are mainly interested in the case of QCD we insert the numerical values of the colour factors and evaluate $\delta Z_{2}^{(4)}$ for $N_{c}=3$. To obtain the corresponding expression we choose $N_{c}=$ 3 after inserting the master integrals but before combining the uncertainties from the various $\varepsilon$ expansion coefficients of the colour factors. Our results for the various powers of $n_{l}$ read [18]

\begin{tabular}{c|c|c|c|c|c}
\hline$\xi=0$ & $1 / \varepsilon^{4}$ & $1 / \varepsilon^{3}$ & $1 / \varepsilon^{2}$ & $1 / \varepsilon$ & $\varepsilon^{0}$ \\
\hline$n_{l}^{0}$ & $-1.77242 \pm 0.00040$ & $-27.6674 \pm 0.0041$ & $-317.093 \pm 0.029$ & $-3142.15 \pm 0.33$ & $-28709.9 \pm 3.2$ \\
$n_{l}^{1}$ & $0.460936 \pm 0.000016$ & $6.69143 \pm 0.00023$ & $74.6540 \pm 0.0013$ & $696.6612 \pm 0.0076$ & $6174.290 \pm 0.084$ \\
$n_{l}^{2}$ & -0.039931 & -0.51572 & -5.5055 & -48.777 & -418.93 \\
$n_{l}^{3}$ & 0.00115741 & 0.0125386 & 0.126757 & 1.07105 & 8.9160 \\
\hline
\end{tabular}

The results for the individual colour structures and higher orders in the gauge parameter $\xi$ are listed in Ref. [18]. Finally, we discuss the wave function renormalization for QED. It is obtained from the QCD result by adopting the following values for the QCD colour factors

$$
C_{F} \rightarrow 1, \quad C_{A} \rightarrow 0, \quad T \rightarrow 1, \quad d_{F}^{a b c d} \rightarrow 1, \quad d_{A}^{a b c d} \rightarrow 0, \quad N_{c} \rightarrow 1 .
$$

We furthermore set $n_{h}=1$ but keep the dependence on $n_{l}$. Note that $n_{l}=0$ corresponds to the case of a massive electron and $n_{l}=1$ would describe the case of the muon with massless electron. Our results read 


\begin{tabular}{c|c|c|c|c|c}
\hline & $1 / \varepsilon^{4}$ & $1 / \varepsilon^{3}$ & $1 / \varepsilon^{2}$ & $1 / \varepsilon$ & $\varepsilon^{0}$ \\
\hline$n_{l}^{0}$ & $0.20500 \pm 0.00037$ & $0.5980 \pm 0.0027$ & $-0.895 \pm 0.021$ & $-6.18 \pm 0.17$ & $-17.4 \pm 1.6$ \\
$n_{l}^{1}$ & $0.17058 \pm 0.00011$ & $0.9556 \pm 0.0014$ & $2.9397 \pm 0.0079$ & $10.480 \pm 0.064$ & $25.92 \pm 0.80$ \\
$n_{l}^{2}$ & 0.056424 & 0.46123 & 3.03509 & 18.7456 & 105.069 \\
$n_{l}^{3}$ & 0.0069444 & 0.075231 & 0.76054 & 6.4263 & 53.496 \\
\hline
\end{tabular}

We have explicitly verified that the QED result is gauge independent after adding the mass counterterm contributions.

\section{Muon anomalous magnetic moment}

In this section we briefly recapitulate our contributions to the calculation of the anomalous magnetic moment of the muon at four loop order and compare with results from other groups. The calculation of the universal contributions is technically very similar to the calculation of the renormalization constants in the on-shell scheme described in the previous sections. In particular, it leads to a subset of the master integrals required there. We briefly discuss our result for the universal contribution obtained in Ref. [19] and only summarize the non-universal ones.

The universal part to $a_{\mu}$ consists of the pure photon contribution and the contribution with closed muon loops. It can be subdivided into six gauge invariant subsets; a representative diagram for each one is shown in the first column of Tab. 1. The second column in Tab. 1 contains the corresponding results from Ref. [20], our work [19], and Ref. [21], respectively (from top to bottom). The results from Ref. [20] are taken from Table I of that reference and the uncertainties are added in quadrature in case several contributions had to be combined. The uncertainties of the results obtained in our work are the quadratically combined results from the individual $\varepsilon$ coefficients of the master integrals. We refrain from introducing a "security factor" (as, e.g., in Ref. [13]) for the universal contribution since the four-loop result for $a_{\mu}$ has also been computed by two other groups. There is no uncertainty in the result provided in Ref. [21].

Within the given uncertainties the results from [20] and our work [19] agree with the semianalytic expressions of [21]. In most cases our uncertainty is at the per cent level or below, except for the contribution in the second row where a $40 \%$ uncertainty is observed. Note, that the absolute size of the uncertainty is of the same order as the one in the first and third row. However, due to cancellations from individual contributions, the central value is significantly smaller.

In the following we summarize the complete four-loop QED contributions and compare the results from the different groups. Denoting the coefficient of $(\alpha / \pi)^{4}$ by $a_{\mu}^{(8)}$ we have

$$
\begin{array}{cccl}
\text { universal } & e^{-} & \tau & e^{-}+\tau \\
a_{\mu}^{(8)}=-1.87(12) & +132.86(48) & +0.0424941(53)+0.062722(10) & {[19,22,23,24]} \\
a_{\mu}^{(8)}=-1.91298(84)+132.6852(60)+0.04234(12) & +0.06272(4) & {[25]} \\
a_{\mu}^{(8)}=-1.9122457649264 \ldots &
\end{array}
$$

Note that the uncertainties in the first line in the parts involving a tau lepton are due to the lepton 


\begin{tabular}{|c|c|}
\hline \multicolumn{2}{|l|}{$\begin{array}{c}\text { Representative } \\
\text { Feynman diagram }\end{array}$} \\
\hline & $-2.1755 \pm 0.0020$ \\
\hline & $-2.161 \pm 0.065$ \\
\hline & -2.176866027739540077443259355895893938670 \\
\hline & $0.05596 \pm 0.0001$ \\
\hline & $0.077 \pm 0.031$ \\
\hline & 0.056110899897828364831469274418908842233 \\
\hline & $-0.3162 \pm 0.0002$ \\
\hline & $-0.3048 \pm 0.021$ \\
\hline & -0.316538390648940158843260382381513284828 \\
\hline & $-0.074665 \pm 0.000006$ \\
\hline & $-0.07461 \pm 0.00008$ \\
\hline & -0.074671184326105513860159965722793126809 \\
\hline & $0.598838 \pm 0.000019$ \\
\hline & $0.597204 \pm 0.0012$ \\
\hline & 0.598842072031421820464649513201747727836 \\
\hline & 0.000876865858889990697913748939713726165 \\
\hline & 0.000876865858889990697913748939713726165 \\
\hline & 0.000876865858889990697913748939713726165 \\
\hline
\end{tabular}

Table 1: The three numbers given in each row (from top to bottom) are taken from [20], our work [19], and [21], respectively.

masses only. After multiplication with $(\alpha / \pi)^{4}$ we obtain for the three equations

$$
\begin{array}{ll}
(-5.44(35)+386.77(1.40)+0.12371(15)+0.182592(29)) \times 10^{-11} & {[19,22,23,24]} \\
(-5.56894(245)+386.264(17)+0.12326(35)+0.18259(12)) \times 10^{-11} & {[25]} \\
(-5.56679893738506 \ldots+\ldots) \times 10^{-11} & {[21]}
\end{array}
$$

The uncertainty of our result is about two orders of magnitudes larger than [25]. It is nevertheless much smaller than the current and foreseen uncertainties from both experiment and the hadronic contributions. This can be seen by considering the difference between the experimental result and the Standard Model prediction which is given by (see, e.g., Ref. [25])

$$
a_{\mu}(\exp )-a_{\mu}(\mathrm{SM}) \approx 250(90) \times 10^{-11} .
$$

The uncertainty is about two orders of magnitude larger than our numerical uncertainty cited above. This remains even true after applying the improvements by a factor 4 . Thus, it can be claimed that the four-loop contribution for $a_{\mu}$ is cross-checked: There are three independent calculations for the universal part and the electron and tau contributions have been computed by two independent groups.

Let us finally remark on $a_{e}$. The Standard Model prediction given in Ref. [21] reads

$$
a_{e}(\mathrm{SM})=115965218.1664(23)(16)(763) \times 10^{-11},
$$


where the three uncertainties have their origin in the numerical accuracy of the five-loop calculation, the hadronic and electroweak corrections and the fine structure constant. Due to the result of Ref. [21] an additional uncertainty of "(60)", which is still present in [20], has been removed. Note that our result for the universal part of $a_{\mu}$ can also be applied to $a_{e}$. However, since it has an uncertainty which is two orders of magnitude larger than the one cited in [20] it is not competitive to [20] and [21].

\section{Conclusions}

We have presented recently obtained results for the renormalization constants of QCD in the on-shell scheme at four-loop order completing the renormalization program at this order in perturbation theory.

Furthermore, we have reviewed the status of calculations of the lepton anomalous magnetic moments comparing results from different groups. All contributions to the anomalous magnetic moments at four-loop order in QED have now been calculated and confirmed by at least two independent groups and methods.

\section{References}

[1] P. Nogueira, J. Comput. Phys. 105 (1993) 279. doi:10.1006/jcph.1993.1074

[2] R. Harlander, T. Seidensticker and M. Steinhauser, Phys. Lett. B 426 (1998) 125 doi:10.1016/S0370-2693(98)00220-2 [hep-ph/9712228].

[3] T. Seidensticker, hep-ph/9905298.

[4] J. A. M. Vermaseren, math-ph/0010025.

[5] J. Kuipers, T. Ueda, J. A. M. Vermaseren and J. Vollinga, Comput. Phys. Commun. 184 (2013) 1453 doi:10.1016/j.cpc.2012.12.028 [arXiv:1203.6543 [cs.SC]].

[6] A. V. Smirnov, JHEP 0810 (2008) 107 doi:10.1088/1126-6708/2008/10/107 [arXiv:0807.3243 [hep-ph]].

[7] A. V. Smirnov and V. A. Smirnov, Comput. Phys. Commun. 184 (2013) 2820 doi:10.1016/j.cpc.2013.06.016 [arXiv:1302.5885 [hep-ph]].

[8] A. V. Smirnov, Comput. Phys. Commun. 189 (2015) 182 doi:10.1016/j.cpc.2014.11.024 [arXiv:1408.2372 [hep-ph]].

[9] P. Marquard, D. Seidel, unpublished.

[10] A. V. Smirnov and M. N. Tentyukov, Comput. Phys. Commun. 180 (2009) 735 doi:10.1016/j.cpc.2008.11.006 [arXiv:0807.4129 [hep-ph]].

[11] A. V. Smirnov, V. A. Smirnov and M. Tentyukov, Comput. Phys. Commun. 182 (2011) 790 doi:10.1016/j.cpc.2010.11.025 [arXiv:0912.0158 [hep-ph]].

[12] A. V. Smirnov, Comput. Phys. Commun. 185 (2014) 2090 doi:10.1016/j.cpc.2014.03.015 [arXiv:1312.3186 [hep-ph]].

[13] P. Marquard, A. V. Smirnov, V. A. Smirnov, M. Steinhauser and D. Wellmann, Phys. Rev. D 94 (2016) no.7, 074025 doi:10.1103/PhysRevD.94.074025 [arXiv:1606.06754 [hep-ph]]. 
[14] P. Marquard, A. V. Smirnov, V. A. Smirnov and M. Steinhauser, PoS RADCOR 2015 (2016) 094 [arXiv:1601.03748 [hep-ph]].

[15] M. Beneke, P. Marquard, P. Nason and M. Steinhauser, Phys. Lett. B 775 (2017) 63 doi:10.1016/j.physletb.2017.10.054 [arXiv:1605.03609 [hep-ph]].

[16] A. H. Hoang, C. Lepenik and M. Preisser, JHEP 1709 (2017) 099 doi:10.1007/JHEP09(2017)099 [arXiv:1706.08526 [hep-ph]].

[17] M. Beneke, Phys. Lett. B 434 (1998) 115 doi:10.1016/S0370-2693(98)00741-2 [hep-ph/9804241].

[18] P. Marquard, A. V. Smirnov, V. A. Smirnov and M. Steinhauser, arXiv:1801.08292 [hep-ph].

[19] P. Marquard, A. V. Smirnov, V. A. Smirnov, M. Steinhauser and D. Wellmann, arXiv:1708.07138 [hep-ph].

[20] T. Aoyama, M. Hayakawa, T. Kinoshita and M. Nio, Phys. Rev. Lett. 109 (2012) 111807 doi:10.1103/PhysRevLett.109.111807 [arXiv:1205.5368 [hep-ph]].

[21] S. Laporta, Phys. Lett. B 772 (2017) 232 doi:10.1016/j.physletb.2017.06.056 [arXiv:1704.06996 [hep-ph]].

[22] A. Kurz, T. Liu, P. Marquard and M. Steinhauser, Nucl. Phys. B 879 (2014) 1 doi:10.1016/j.nuclphysb.2013.11.018 [arXiv:1311.2471 [hep-ph]].

[23] A. Kurz, T. Liu, P. Marquard, A. V. Smirnov, V. A. Smirnov and M. Steinhauser, Phys. Rev. D 92 (2015) no.7, 073019 doi:10.1103/PhysRevD.92.073019 [arXiv:1508.00901 [hep-ph]].

[24] A. Kurz, T. Liu, P. Marquard, A. Smirnov, V. Smirnov and M. Steinhauser, Phys. Rev. D 93 (2016) no.5, 053017 doi:10.1103/PhysRevD.93.053017 [arXiv:1602.02785 [hep-ph]].

[25] T. Aoyama, M. Hayakawa, T. Kinoshita and M. Nio, Phys. Rev. Lett. 109 (2012) 111808 doi:10.1103/PhysRevLett.109.111808 [arXiv:1205.5370 [hep-ph]]. 